\title{
BMJ Open Conceptualisation and measurement of adaptation within the Roy adaptation model in chronic care: a scoping review protocol
}

\author{
Xiyi Wang (D) , ${ }^{1}$ Qi Zhang, ${ }^{1}$ Jing Shao, ${ }^{2}$ Zhihong Ye (D) ${ }^{1}$
}

To cite: Wang $X$, Zhang $Q$, Shao J, et al. Conceptualisation and measurement of adaptation within the Roy adaptation model in chronic care: a scoping review protocol. BMJ Open 2020;10:e036546. doi:10.1136/ bmjopen-2019-036546

- Prepublication history for this paper is available online. To view these files, please visit the journal online (http://dx.doi. org/10.1136/bmjopen-2019036546).

Received 19 December 2019 Revised 22 April 2020 Accepted 27 May 2020
Check for updates

(c) Author(s) (or their employer(s)) 2020. Re-use permitted under CC BY-NC. No commercial re-use. See rights and permissions. Published by BMJ.

${ }^{1}$ Department of Nursing, Zhejiang University School of Medicine Sir Run Run Shaw Hospital, Hangzhou, Zhejiang, China

${ }^{2}$ Department of Nursing, Zhejiang University School of Medicine, Hangzhou, Zhejiang, China

Correspondence to

Dr Zhihong Ye; yezh@zju.edu.cn

\section{ABSTRACT}

Introduction The Roy adaptation model provides a basis for developing the science of nursing. Its theoretical assumptions have been tested in empirical studies. Although several works have historically reviewed the development of this model, a refinement of its key concepts is needed. The proposed scoping review aims to describe how the concept of adaptation was defined and measured in nursing studies related to chronic health conditions.

Methods and analysis This scoping review will adopt the methodology proposed by Arksey and O'Malley. Several databases, including MEDLINE (OVID), CINAHL, EMBASE, PsycINFO, PubMed, Wan Fang, China National Knowledge Infrastructure and VIP net, will be selected and used to mine literature published in English and Chinese languages, up to December 2019. Key terms related to 'Roy adaptation model' will be identified and used for developing tailored search strategies for each database. Articles will be included in the analysis if they are primary research reports explaining the concept of adaptation within the field of chronic care. All screening and extraction of literature will be independently performed and checked by two authors, according to the guideline of Preferred Reporting Items for Systematic Review and Meta-Analysis-Extension for Scoping Reviews. The findings will be organised and summarised into narratives in line with the construction of conceptual-theoreticalempirical system of knowledge for further consultation and translation.

Ethics and dissemination This scoping review does not require ethical approval. The findings are expected to be published in peer-reviewed English or Chinese journals as well as conference proceedings in the area of chronic care.

\section{INTRODUCTION}

Non-communicable diseases (NCDs), mainly cardiovascular diseases, cancers, chronic respiratory diseases and diabetes, result from a combination of genetic, physiological, environmental as well as behavioural factors. ${ }^{1}$ WHO has predicted that more than 14 million people will die prematurely from

\section{Strengths and limitations of this study}

- This is the first scoping review to synthesise the Roy adaptation model-based research according to the conceptual-theoretical-empirical structure in chronic care.

- The findings of this study are expected to provide an evaluation of health outcomes with a holistic view, using the concept of adaptation by describing the relationship between human, environment and health.

- This review will include studies published in English and Chinese languages.

- One limitation of this review is the potential to miss out on relevant articles, especially experts' opinions and summaries on the definition of adaptation.

- Another limitation may arise from the restriction of the search language.

NCDs between the age of 30 and $70(\mathrm{p} 1){ }^{2}$ Patients with NCDs require prolonged and repeated treatments, which necessitates a lifetime of symptoms monitoring, exposure to various medication regimens as well as behaviour modification. ${ }^{3-5}$ Additionally, some of the NCDs and the associated treatments may lead to complications. Identification and management of risk factors of diseases is a demanding process that results in stress. In the context of NCDs, stress is defined in terms of the internal state, external event and experience from a person-environment interaction. ${ }^{6}$ An individual's ability to effectively deal with stressful events can be defined as coping. ${ }^{7}$ Furthermore, the challenges of living with a chronic illness drive a person to find new ways of coping to adapt to altered health conditions. Previous studies demonstrated that chronic diseases are associated with a complex, unpredictable, longterm and continuously evolving course. ${ }^{389}$ A number of social-political, health services and cultural factors also affect the ability of people to cope with NCDs. ${ }^{4810}$ 
The use of the term adaptation to delineate human responses to chronic health conditions across physiological, psychological and social domains has gained traction. ${ }^{9-11}$ Roy ${ }^{12}$ defined the adaptation as the process and outcome by which the thinking and feeling person uses conscious awareness and choice to create human and environmental integrations. Elsewhere, a study by Biesecker $e t$ at described adaptation as the dynamic and multidimensional process of dealing with health threats as well as the outcomes of that process. Adaptation is a match between the demands of a situation and capacities of an individual in psychosocial and biomedical models. ${ }^{6}$ Several lines of thought suggest that adaptation can be viewed as a process that reflects an individual's efforts to regulate interactions with others and the environment as well as an outcome that presents a state of physical, emotional, behavioural and cognitive functions. Therefore, conceptualisation of adaptation, according to derived theory development, as well as inductive and deductive empirical strategies are the basis for developing an implementable theory, practical instruments and intervention strategies.

Coping theories help to gain insights into components used for constructing adaptation. For example, the coping model proposed by Lazarus ${ }^{13}$ revealed an association between stress appraisals and coping strategies. Moreover, coping affects adaptation in complicated ways. The Roy adaptation model (RAM) outlines the theoretical concepts of stimuli, coping process, adaptive modes such as physical, self-concept, role function and interdependence as well as adaptive response including adaptive or ineffective behaviours (table 1). ${ }^{14}{ }^{15}$ RAM explores the interrelationships among humanism, health and the environment. Further explanation of adaptation was presented in the perspectives of information processing such as inputs, control processes, outputs and feedback. ${ }^{15}$ However, numerous unanswered questions remain with regards to how conceptualise and measure adaptation.

Over the past 50 years, RAM has been widely used as a conceptual model to guide education, research, practice and knowledge development. The Boston-based adaptation research in nursing society, currently known as Roy Adaptation Association (RAA), established to develop RAM, conducted and published a thorough critique and integration of RAM-based researches (1970-1995). ${ }^{16}$ Thereafter, Roy ${ }^{18}$ published the examination and synthesis of findings from a large body of RAM research in 2011. A review by Dobratz ${ }^{18}$ provides evidence that supports the propositions and conceptualisations of adaptation. Additionally, critical interpretive syntheses of qualitative research were performed, between 1995 and 2005 , to illustrate ways and contexts in the development of RAM. ${ }^{19}$ Overall, these secondary analyses of RAMbased researches were organised by the core elements of RAM but were not focused on the field of chronic illness.

The adaptation proposed in RAM requires more explicit explanations at an operational level. Consequently, various middle-range theories were developed for different nursing situations to address the adaptation with concrete definitions and limited variables. ${ }^{18}$ However, gaps remain between theory and practice in nursing contexts. For example, Pollock ${ }^{8}$ established a theory of adaptation to chronic illness, which was tested

\section{Table 1 The core elements of the RAM}

\begin{tabular}{|c|c|}
\hline Concepts & Definitions \\
\hline Stimuli & $\begin{array}{l}\text { That which provokes a response, or more generally, the point of interaction of the human } \\
\text { system and environment. }\end{array}$ \\
\hline Adaptive response & Responses that promote integrity in terms of the goals of human systems. \\
\hline Physiologic mode & $\begin{array}{l}\text { An individual manifests the physical and chemical processes involved in the function and } \\
\text { activities of a living organism. }\end{array}$ \\
\hline Self-concept mode & $\begin{array}{l}\text { The composite of belief and feelings that is held about oneself at a given time, formed from } \\
\text { internal perception and perceptions of others' reactions. }\end{array}$ \\
\hline Group identity mode & $\begin{array}{l}\text { Shared relations, goals and values, which create a social milieu and culture, a group self- } \\
\text { image and coresponsibility for goal achievement. }\end{array}$ \\
\hline Interdependence (individual) & $\begin{array}{l}\text { The close relationships of people aimed at satisfying needs for affection and the development } \\
\text { of relationships. }\end{array}$ \\
\hline Interdependence (group) & The dynamic interrelationships of system of people. \\
\hline
\end{tabular}

RAM, Roy adaptation model. 
using five empirical studies. Although this theory was well developed, health-related variables and assessment tools used for measuring concepts differed among studies, leading to potential difficulties in synthesising empirical data across studies. A subsequent review conducted to evaluate the tools used to assess the core elements of RAM found that no instrument was effective in conceptualising and measuring individuals as adaptive systems. ${ }^{20}$ A meta-analysis by Chiou ${ }^{14}$ evaluated empirical adequacy of the interrelationships between adaptive modes (physical, self-concept, role function and interdependence) in RAM. Results from nine studies revealed small to medium size effects between every two modes, with nonsignificant effects between interdependence and physiological modes. However, it is still imperative to verify the credibility of RAM displayed by the relationships in all adaptive modes, based on homogeneous qualitative or quantitative studies. A review by Cai and $\mathrm{CaO}^{21}$ described the understanding and applications of self-concept mode in chronic care. However, most of the studies focusing on adaptation and RAM do not adequately discuss the operational definition of adaptation. Moreover, the prevailing literature does not provide detailed clarification and effective measurement of adaptation in chronic care.

Globally, several literature reviews have been published in English and Spanish. ${ }^{1722}$ These reviews show that RAM has been tested within the western culture, but little is known regarding the application of the assumptions and alignment of RAM in the content of Chinese culture (ie, God). Chinese people have been taught and are actively influenced by Confucianism and Taoism views of a person's links to the world. For instance, individuals tend to keep a balance and avoid extreme behaviours. Consequently, they have learnt to live in harmony with the universe. This vision is in line with Roy's philosophical assumptions that people have mutual relationships with the world and a God-figure, ${ }^{12}$ therefore make it possible to apply RAM in Chinese clinical settings. Over 300 articles related to RAM have been published in Chinese journals, between 2000 and 2016. ${ }^{23}$ However, no critical analysis of issues of content consistency and methods has been reported in such studies. It is therefore important to test the propositions of RAM by conducting an in-depth analysis of studies published in the Chinese language. Estimates of RAM-based research productivity, quality of empirical evidence and synthesis of research findings remain unclear, necessitating further refinement.

In this paper, we formulate a protocol for a scoping review to provide an overview of the RAM as a conceptual model for chronic care. This review is expected to map literature on the operationalisation of adaptation at the theoretical level and to aid in exploring assessment tools for adaptation measures in empirical work. We formulate the following objectives for the review, based on the underlying tenets of the RAM and conceptual-theoretical-empirical structure: (1) describe the scope, such as health fields, targeted populations and topics addressed, (2) synthesise the operational definitions for clarifying adaptation, (3) summarise the characteristics and variables for identifying adaptation, (4) conclude the dimensions and indicators for measuring adaptation in chronic care and (5) identify the gaps in evidence and areas for further inquiry. A greater understanding of the concept of adaptation, which determines patients' health status and outcomes, may lead to the exploration of adaptive responses and optimisation of behaviours that promote health.

\section{METHODS AND ANALYSIS}

Although several studies have explored the RAM, the existing knowledge is heterogeneous and inconclusive due to the complicated nature of adaptation within the RAM. Based on this, the methodology of the scoping review (proposed by Arksey and O'Malley ${ }^{24}$ and advanced by Levac et $a l^{25}$ ) is appropriate for use in this study. This systematic scoping review has six stages: (1) identification of the research question, (2) identification of relevant studies, while balancing feasibility with breadth and comprehensiveness of the scoping process, (3) selection of eligible studies, which involves using an iterative team approach, (4) charting the data by incorporating numerical summary and narrative synthesis, (5) collating, summarising and reporting the results, including identifying the implications of the study findings and (6) consulting and translating knowledge. This protocol follows the Preferred Reporting Items for Systematic Review and Meta-Analysis (PRISMA) Protocol statement. ${ }^{26}$

\section{Stage 1: identification of the research question}

According to the core concepts organised by the extended population, concept and context (PCC), our research questions are as follows:

1. What chronic health conditions (such as diabetes, heart failure, hypertension, cancer) and populations (individual or group, patients, informal caregivers and healthcare professionals) have been targeted for generating and testing the theory? (refers to objective 1).

2. What core RAM elements are used to identify the concept of adaptation? (refers to objectives 2 and 3 ).

3 . What conceptual-theoretical-empirical frameworks have been derived from the RAM for testing and practice? (refers to objectives 2 and 3 ).

4. What health-related variables (physiological, selfconcept, role function and interdependence modes) are frequently used to identify adaptation in chronic care? (refers to objectives 2 and 3 ).

5 . What empirical indicators (such as symptoms, quality of life, treatment adherence, social support, autonomy, engagement of decision-making, self-efficacy, reconstruction of a new self) are examined to make the adaptation operationalisable? (refers to objectives 3 and 4).

6. What kinds of instruments (such as capacities of coping strategies, perceptions of illness and disease-specific quality of life) have been selected to determine the 
adaptive responses or maladaptive responses? (refers to objective 4).

\section{Stage 2: identification of relevant studies}

Before attempting to scope the RAM-based research, a well-defined 'adaptation' should be clarified. This scoping review will build on Roy's definition of adaptation and the core elements of RAM. Definitions of stimuli, the coping process, adaptive response and four adaptive (physiological/physical, self-concept/group identity, role function and interdependence) modes are outlined in (table 1) ${ }^{15}$ Consistent with WHO's definition, Corbin and Strauss ${ }^{27}$ described chronic illness as serious diseases that could last a person's entire life affecting their mental, emotional and social well-being. Although no axiomatic definition has been agreed on for chronic diseases, there are similar attributes, including long latency, protracted clinical course, uncertain aetiology and no definitive 'cure'. ${ }^{28}$

In the initial search of the databases for published systematic or scoping reviews, no reviews on this topic were identified. To review the literature related to RAMbased research in chronic care, a systematic search will be conducted. Electronic databases, MEDLINE (OVID), CINAHL, EMBASE, PsycINFO and PubMed, will be searched to identify English-language publications. In addition, Wan Fang Database, China National Knowledge Infrastructure and VIP net will be scrutinised to identify publications in Chinese language. Grey literature, mainly information generated by the RAA and a series of conference proceedings, will further be identified using accessible approaches. No attempts will be made to contact authors for further information during the literature search. All literature searches will be conducted by two trained researchers, who will use the following keywords to access publications: 'Roy adaptation model' for English and '罗伊适应模式 (Roy adaptation model)' for Chinese databases. The search strategies will be tailored according to the command language used by each database (such as Emtree terms, CIHNAHL headings and the use of text words). The search strategies for different databases will be reviewed by librarians at the University of Toronto and Zhejiang University. The search will be limited to studies involving human subjects, and articles published either in English or Chinese between January 1970 and December 2019, with 1970 set as a time point when RAM was established and published by Roy. ${ }^{12}$ To ensure that data are relevant to theory description and testing, this scoping review will consider both qualitative and quantitative studies. Specifically, we will enrol experimental and quasi-experimental study designs including randomised controlled, non-randomised controlled trials and interrupted time-series studies. Systematic and scoping reviews will be excluded after reading the title and abstract. Reference lists will be reviewed to retrieve related primary studies to hand search for further studies. Based on a pilot search we conducted, the following RAM-related terms were used in PubMed:
$(((($ English [Language] $) \quad$ OR Chinese[Language $]))$ AND $(((()(()((()(($ 'nursing theory') AND Roy $))$ OR (('nursing theory') AND adaptation)) OR (('conceptual model') AND Roy)) OR (('nursing model') AND Roy)) OR (('nursing model') AND adaptation)) OR 'Roy's adaptation model') OR ('adaptation model' and nursing)) OR ('Roy's theory' and adaptation)) OR ('Roy theory' and adaptation)) OR ('Roy theory' and nursing)) OR ('Roy model' and nursing)) OR ('Roy model' and adaptation)) OR 'Roy adaptation model')) AND ('1970' [Date - Publication] : '2019' [Date - Publication]).

The search resulted in 798 studies, related to RAM, based on the title and abstract screening. Note that the terms of chronic diseases will not be used in literature search due to the manageable number of searched literature and wide range of chronic illness. Identification of RAM-related literature in chronic care will be conducted at the study selection phase.

\section{Stage 3: study selection}

To standardise the study selection process, we will follow the PRISMA-Extension for Scoping Review guidelines. ${ }^{29}$ Briefly, a two-step process will be employed for study selection and performed independently by two reviewers (XW and QZ). The bibliographic information of the included articles will be amalgamated and stored using Endnote V.X8. In the screening of titles and abstracts, reviewers will check whether RAM is involved. Thereafter, they will read the full text applying the eligibility criteria outlined below. The studies to be included should be agreed by the two reviewers. In case of disagreements, a third reviewer (JS) will be involved for further assessment. If needed, a discussion among all reviewers will be carried out until a consensus is reached. A pilot study, for testing literature search and screening, was developed revealing a consensus among our researchers.

The proposed review will analyse studies that use the concept of adaptation based on the RAM to define the process and outcomes of creating human and environmental integration during the management of chronic health conditions. Participants may exhibit other descriptions of adaptation including, but not limited to, adjustment or self-regulate in conjunction with adaptation. All published and unpublished literature reporting findings on theory development, quantitative, qualitative, mixed or multimethod studies based on the RAM will be included. According to PCC and research questions, the inclusion criteria adapted from RAA include:

1. Studies published in English or Chinese.

2. Studies published as a primary research report.

3. Studies determined to be within the field of human care for chronic diseases.

4. Studies explicitly used the RAM as a conceptual framework to design the study and to interpret the results.

5. Studies clarified the components of adaptation using limited and measurable variables. 
6. Studies in which quantitative, qualitative and mixedmethods were designed to describe the adaptation in different practice settings.

\section{Stage 4: data extraction and charting the data}

When the final set of articles are included, a confirmation from the research team should be obtained before data are extracted into an evidence table. In this proposed scoping review, data extraction and charting of the results will be iterative process. Key information to be recorded from the sources will include authors, year of publication, origin, purpose, study design, study population, methods, theoretical framework, measurable indicators, assessment tools and key findings related to the questions. These should be aligned with the research objectives and contribute to knowledge synthesis. Specifically, theoretical frameworks may point out the theory basis at the operational level; measurable indicators may demonstrate the effective evidenced-based individual strategies and approaches in the adaptation process; assessment tools may be used for measuring components of adaptation; key findings may reveal how adaptation is understood in chronic care and describe the gaps. When analysing the types of study, glossary terms set by the RAA will be used (p29 and 30). ${ }^{30}$ Quantitative studies include descriptive, explain-predict-prescribe, intervention studies, whereas qualitative ones employ methods of content analysis, grounded theory and phenomenology.

Selected studies, that meet inclusion criteria, will be imported into NVivo V.12.0 software for coding, then a combined qualitative and quantitative content analysis will be applied. First, initial ideas related to study objectives will be noted down, for instance, the variables and indicators used in empirical studies for measuring adaptation. Second, a description of these data will be scrutinised in detail and identified as meaningful segments, before codes are generated. Third, different codes will be collected into predefined categories according to the terms shown in table 1. Finally, other extracts will be reviewed and summarised for correlation analysis.

The key information chosen by reviewers for the chart may be further redefined at the review stage, depending on the literature included. All included literature will be independently reviewed, analysed and charted into tables by two reviewers. Quality assessment for each study and metaanalysis of quantitative results will not be considered in this scoping review because they are outside the study objectives. So far, a pilot extraction process from seven studies by two reviewers (XW and QZ) has generated a consensus.

\section{Stage 5: collating, summarising and reporting results}

A PRISMA diagram will be developed to outline the process and results of the study search, selection and analysis. To clearly and systematically present the review results, two strategies will be used: (1) a numerical overview of the number, type and distribution of included studies and (2) a narrative synthesis of conceptualisation and measurement of adaptation in chronic care.
Despite the identification of many potential strategies for the literature review, narrative synthesis, ${ }^{31}{ }^{32}$ which primarily relies on the use of words and text, will be employed to synthesise the empirical evidence relevant to research questions. The identified, extracted health-related variables and empirical indicators will be coded based on RAM, then the codes with their detailed descriptions sorted to fit predefined categories.

Objective 1: the scope of the RAM-based research can be narrowed down to specific diseases and populations in chronic care. We will use counts and then determine how many studies focused on each research topic. The characteristics of the study, including its purpose, population, addressed theory concepts and findings that contributed to the RAM, will be illustrated.

Objective 2: for operational definitions relating to adaptation, we will use qualitative content analysis to create a content summary of theory-generating research. The theoretical structure of the RAM, including middle-range theory and specific-situation theory, can interpret adaptation in terms of environmental stimuli, coping processes and four adaptive responses by providing relatively concrete concepts and statements. A textual summary table for data from each study will be created to show theory concepts, theory assumptions, the linkage to the RAM and implications for chronic care.

Objective 3: concerning health-related variables used for identifying the adaptation, we will analyse theory-testing research aimed at explaining theoretical concepts. Core elements of the RAM, such as stimuli, coping processes and four adaptive modes, will be used to categorise the identified studies into different tables. Then, descriptive statistics of generated codes will be displayed. Using findings from content analysis, each table will show operationalisation of adaptation in nursing research and practice, thereby contributing to refinement of the RAM.

Objective 4: coded empirical indicators will be tabulated based on the predefined categories (table 1). The instruments and their consistency with RAM concepts will be assessed and summarised accordingly.

Objective 5: the overview of conceptual-theoretical-empirical structure of the RAM can be obtained by analysing a large body of the literature. We expect to generate summary tables for numerical and narrative variables to show the construction of adaptation, including stimuli, coping processes, adaptive responses and outcomes. In addition, the links between existing studies and the RAM will be identified. More importantly, we will highlight empirical evidence with profound implication for future research on adaptation and evidence-based chronic care practice.

\section{Stage 6: consultation and knowledge translation}

To maintain logical consistency and uniformity in the RAM, we will hold consultations with the nursing theorist Roy or other RAA experts through email for a confirmation. Before that, we will present results from our preliminary analysis of adaptation conceptualisation using well-structured tables and questions. To improve the cross-cultural scope of the 
study, we will provide an overview of RAM-guided research within Chinese culture. In addition, a preliminary comparison of content consistency between studies in Chinese and those in the English language may be outlined.

Stakeholder consultation can provide insights into what is reported in the literature. ${ }^{25}$ In this study, we will share our findings with 5-10 nursing researchers who have expertise in healthcare through monthly seminars. We will conduct a series of face-to-face or email consultations with key informants and take detailed notes as well as summaries of their feedbacks. These results are expected to contribute to our narrative synthesis and stimulate the prospect of establishing a middle-range theory of adapting to chronic illness. More importantly, directions for clinical practice will be settled. In addition, we hope to further develop and implement interventions for promoting individuals' adaptive behaviours in the physiologic, self-concept, role function and interdependence modes. If applicable, we will test the effectiveness of these interventions among patients with chronic illness, following ethical approval. Furthermore, we may make an outline of interviews for patients and their caregivers, based on the construction of adaptation within the RAM. Empirical works will be used to validate the knowledge acquired from this scoping review.

\section{PATIENT AND PUBLIC INVOLVEMENT}

Patients and the public were not involved in the design, or conduct, or reporting or dissemination of this protocol.

\section{ETHICS AND DISSEMINATION}

Ethics approval is neither required nor applicable to this scoping review. Findings from the study are expected to contribute to team members' (XW and QZ) thesis. Highlights and findings herein will be submitted for publication in peer-reviewed journals and conferences in the areas of nursing, public health and health service to advocate for the integration of adaptation research during the management of chronic diseases. Depending on the findings, there could be a need for a subanalysis to enable exploration of adaptation for a specific chronic condition. This may take into consideration different individuals' adaptive challenges, responses, tasks and strategies. If sufficient literature is identified and retrieved, our research team will plan for a systematic review or meta-analysis. The results of this scoping review will, therefore, provide a foundation for further studies on the development of instruments and nursing interventions to facilitate peoples' adaptation.

Acknowledgements The authors wish to thank Professor Doris Howell of the University of Toronto for her professional suggestions on developing the scoping review.

Contributors XW conceived the study and drafted the scoping review protocol under the supervision of ZY; XW and QZ will conduct independent database searches and screening of all titles and abstracts. XW, QZ and JS will be responsible for reviewing the included studies. All authors contributed to the protocol design and ongoing plan.
Funding This work was supported by Department of Health of Zhejiang Province (grant number2019ZD034). XW was supported by China Scholarship Council (grant number201806320175). The funder had no role in the entire review study

Competing interests None declared.

Patient consent for publication Not required.

Provenance and peer review Not commissioned; externally peer reviewed.

Open access This is an open access article distributed in accordance with the Creative Commons Attribution Non Commercial (CC BY-NC 4.0) license, which permits others to distribute, remix, adapt, build upon this work non-commercially, and license their derivative works on different terms, provided the original work is properly cited, appropriate credit is given, any changes made indicated, and the use is non-commercial. See: http://creativecommons.org/licenses/by-nc/4.0/.

\section{ORCID iDs}

Xiyi Wang http://orcid.org/0000-0002-6470-8556

Zhihong Ye http://orcid.org/0000-0001-6947-3330

\section{REFERENCES}

1 WHO. Noncommunication diseases, 2018. Available: https://www. who.int/en/news-room/fact-sheets/detail/noncommunicablediseases [Accessed 21 Apr 2019].

2 WHO. Global action plan for the prevention and control of noncommunication diseases 2013-2020, 2013. Available: https:// apps.who.int/iris/bitstream/handle/10665/94384/9789241506236_ eng.pdf;jsessionid=86D38232C96851F6B0A84F4C6EF9E0C5? sequence $=1$ [Accessed 21 Apr 2019].

3 de Ridder D, Geenen R, Kuijer R, et al. Psychological adjustment to chronic disease. The Lancet 2008;372:246-55.

4 Ambrosio L, Senosiain García JM, Riverol Fernández M, et al. Living with chronic illness in adults: a concept analysis. J Clin Nurs 2015;24:2357-67.

5 Riegel B, Jaarsma T, Lee CS, et al. Integrating symptoms into the middle-range theory of self-care of chronic illness. ANS Adv Nurs Sci 2018.

6 Aldwin CM. Stress, coping, and developement an integrative perspective. New York: The Guilford Press, 2007.

7 Audulv Åsa, Packer T, Hutchinson S, et al. Coping, adapting or selfmanaging - what is the difference? A concept review based on the neurological literature. J Adv Nurs 2016;72:2629-43.

8 Pollock SE. Adaptation to chronic illness: a program of research for testing nursing theory. Nurs Sci Q 1993;6:86-92.

9 Biesecker BB, Erby LH, Woolford S, et al. Development and validation of the psychological adaptation scale (PAS): use in six studies of adaptation to a health condition or risk. Patient Educ Couns 2013;93:248-54.

10 Helgeson VS, Zajdel M. Adjusting to chronic health conditions. Annu Rev Psychol 2017;68:545-71.

11 Whittemore R, Dixon J. Chronic illness: the process of integration. $J$ Clin Nurs 2008;17:177-87.

12 Roy C. Future of the Roy model: challenge to redefine adaptation. Nurs Sci Q 1997;10:42-8.

13 Lazarus RS. Coping theory and research: past, present, and future. Psychosom Med 1993;55:234-47.

14 Chiou CP. A meta-analysis of the interrelationships between the modes in Roy's adaptation model. Nurs Sci Q 2000;13:252-8.

15 Roy C. The Roy adaptation model. Third ed. New Jersey: Pearson Education, Inc, 2009.

16 Boston Based Adaptation Research in Nursing Society. Roy adaptation model-based research: 25 years of contribution to nursing science. Indianapolis Center Nursing Press, 1999.

17 Roy C. Research based on the Roy adaptation model: last 25 years. Nurs Sci Q 2011;24:312-20.

18 Dobratz MC. Moving nursing science forward within the framework of the Roy adaptation model. Nurs Sci Q 2008;21:255-9.

19 Perrett SE. Review of Roy adaptation model-based qualitative research. Nurs Sci Q 2007;20:349-56.

20 Barone SH, Roy CL, Frederickson KC. Instruments used in Roy adaptation model-based research: review, critique, and future directions. Nurs Sci Q 2008;21:353-62.

21 Cai T, Cao M. Application progress on Roy's Adaptation Model in self-concept care of patients with chronic diseases. Chin Nurs Res 2016;30:3991-3.

22 Fawcett J. Using the Roy adaptation model to guide research and/or practice: construction of Conceptual-Theoretical-Empirical systems of knowledge. Aquichan 2009;9:1657-5997. 
23 Pan L, Bao Y. Bibliometric analysis of the literature of Chineselanguage Roy adaptation model-based research from 2000 to 2016. J Tradit Chin Med 2018;26:39-42.

24 Arksey H, O'Malley L. Scoping studies: towards a methodological framework. Int J Soc Res Methodol 2005;8:19-32.

25 Levac D, Colquhoun H, O'Brien KK. Scoping studies: advancing the methodology. Implement Sci 2010:5:69.

26 Shamseer L, Moher D, Clarke M, et al. Preferred reporting items for systematic review and meta-analysis protocols (PRISMA-P) 2015: elaboration and explanation. BMJ 2015;350:97647.

27 Corbin JM, Strauss A. A nursing model for chronic illness management based upon the trajectory framework. Sch Inq Nurs Pract 1991:5:155-75.
28 Rothenberg RB, Koplan JP. Chronic disease in the 1990s. Annu Rev Public Health 1990;11:267-96.

29 Tricco AC, Lillie E, Zarin W, et al. PRISMA extension for scoping reviews (PRISMA-ScR): checklist and explanation. Ann Intern Med 2018;169:467-73.

30 Roy C. Generating middle range theory: from evidence to practice. New York: Springer Publishing Company, 2014.

31 Fawcett J. Thoughts about conceptual models, theories, and literature reviews. Nurs Sci Q 2013;26:285-8.

32 Kastner M, Antony J, Soobiah C, et al. Conceptual recommendations for selecting the most appropriate knowledge synthesis method to answer research questions related to complex evidence. $J$ Clin Epidemiol 2016;73:43-9. 